\title{
Psychometric properties of the Chinese version Fear of Cancer Recurrence Questionnaire-7 (FCR-7)
}

Running title: Psychometric properties of the Chinese FCR-7

\author{
Yuan Yang ${ }^{1 \#}$ MSc, \\ Gerry Humphris ${ }^{2 \#}$ PhD,

1. Department of Psychiatry, Nanfang Hospital, Southern Medical University, Guangdong-Hong Kong-Macao Greater Bay Area Center for Brian Science and Brain-Inspired Intelligence, Guangzhou, Guangdong, China. 2. School of Medicine, University of St Andrews, St Andrews, Scotland, UK. 3 Department of Radiotherapy, Cancer Center, Guangdong Provincial People's Hospital (Guangdong Academy of Medical Sciences), Guangzhou, Guangdong, China. 4. Department of Anatomy, Guangzhou Medical University 5. Department of Radiotherapy, Nanfang Hospital, Southern Medical University, Guangzhou, Guangdong, China.

\# These authors contributed equally to the work.

\section{Correspondence}

Prof Bin Zhang, Department of Psychiatry, Nanfang Hospital, Southern Medical University, Guangzhou, China, 510515, Email: zhang73bin@hotmail.com, Tel/Fax: +86 (020) 61642066. 
Yuan Yang received her MSc in counselling and psychotherapy from the university of Edinburgh, UK. She serves as a clinical psychologist at Department of Psychiatry, Southern Medical University Nanfang Hospital. Her areas of professional interest include psycho-oncology and psychotherapy.

Gerry Humphris received his PhD in psychology from Guy's Hospital, University of London, UK. He is currently a professor of Health Psychology at the University of St Andrews. His areas of professional interest include psycho-oncology, oral health, head and neck cancer and health behavior.

Hengwen Sun received his MD and PhD from the Southern Medical University, China. He is currently a consultant doctor at Department of radiotherapy, Guangdong Provincial People's Hospital. His areas of professional interest include psycho-oncology, breast and stomach cancer.

Wengao Li received his MD from the Southern Medical University, China. He is currently a resident doctor at Department of Psychiatry, Southern Medical University Nanfang Hospital. His areas of professional interest include anxiety, depressive disorder and insomnia.

Yanli Hao received her PhD and MD from the Chinese University of Hongkong. She is now a professor at Department of Anatomy, Guangzhou Medical University. Her areas of professional interest include brain function, cognition in patients with mental disorder.

Ting Liu received her bachelor's degree in nursing from Wuhan Polytechnic University, China. She is now a research nurse at Department of Psychiatry, Southern Medical University Nanfang Hospital. Her areas of professional interest include cancer nursing, and palliative care.

Jingying Zhang received her MSc in psychology from Nanjing Normal University, China. She is now a clinical psychologist at Department of Psychiatry, Southern Medical University Nanfang Hospital. Her areas of professional interest include cognitive behavioral therapy.

Hongmei Wang received her MD and PhD from medical school, Sun Yat-sen University, China. She is currently a consultant doctor at Department of radiotherapy, Southern Medical University Nanfang Hospital. Her areas of professional interest include breast cancer and psycho-oncology.

Bin Zhang received his MD and PhD from the Chinese University of Hongkong. He is currently a professor and chief of the Department of Psychiatry, Southern Medical University Nanfang Hospital. His areas of professional interest include mental illness and insomnia. 


\section{Public Significance Statement}

The present study suggests that the Chinese version Fear of Cancer Recurrence Questionnaire-7 (FCR-7) is a reliable and valid measurement for assessing patient's recurrence fear. Additionally, patients with low income, family cancer history and those who had gone through chemotherapy are more likely to report higher fears. Therefore, specific and flexible psychological interventions are needed for these high-risk populations.

\section{Compliance with Ethical Standards}

\section{Funding Information}

This study is funded by the President Foundation of Nanfang Hospital, Southern Medical University (2017L001); Guangzhou Science and Technology Project (201804010132); Key Item of Guangzhou bureau of education (2019KC106), and Innovation Item of Guangdong Provincial Department of Education (2018A043442).

\section{Conflict of interest}

Authors declare that they have no conflict of interest.

\section{Ethics Approval}

The Southern Medical University Nanfang Hospital Research Ethics Committee examined and approved the study (ref No: NFEC-2018-038).

\section{Acknowledgements}

We sincerely thank all the participants and many colleagues who we have discussed this manuscript with. 


\section{Abstract}

This paper investigates the reliability and validity of the Chinese version of the Fear of Cancer Recurrence Questionnaire-7 (FCR-7). A total of 1025 cancer patients were recruited and asked to complete the Chinese FCR-7, FoP-Q-SF, PHQ-9, and GAD-7. The internal consistency and test-retest reliabilities were examined. EFA and CFA was conducted on random split-half samples. Overall relationships of FCR-7 with other psychological constructs were examined. The Chinese FCR-7 showed good internal consistency (Cronbach's alpha $=0.87)$, test-retest reliability $(r=0.90)$, and item-total correlations (ranged from 0.583 to 0.872 ). The unitary factor structure was supported by the EFA and the CFA fit statistics (CFI = 0.99; RMSEA $=0.039,95 \% \mathrm{Cl}$ : $0.01,0.07)$. The total score of FCR-7 was positively associated with FoP-Q-SF ( $r=$ $0.756, P<.01)$, PHQ-9 $(r=0.522, P<.01)$, and GAD-7 $(r=0.553, P<.01)$. Patients with low monthly income $(P<.001)$, family cancer history $(P=.012)$, and those who had gone through chemotherapy $(P=.001)$ tended to report higher FCR. The FCR-7 has been translated and successfully culturally adapted into a Chinese version. It is a reliable and valid measurement for assessing FCR.

Keywords: cancer, Chinese, Fear of recurrence, psychometric properties Word count: Abstract: 195; Full Text: 3490 


\section{Introduction}

In the recent decade, Fear of Cancer Recurrence (FCR) has received growing attention in research with many cancer survivors of various cancer diagnoses (Cohee et al., 2015). FCR is often defined as: fear, worry, or concern relating to the possibility that cancer will come back or progress (Lebel et al., 2016). Patients with high FCR often reported significant psychological distress (i.e. depression) as well as negative behavior change (i.e. avoidance, excessive self-examination) (Avis et al., 2005; Lasry \& Margolese, 1992; Lebel, Rosberger, Edgar, \& Devins, 2009; Simard, Savard, \& Ivers, 2010). This concern may appear immediately after cancer diagnosis/treatment and has been shown to remain stable for years (Simard \& Savard, 2009).

Recent study showed that about $24-40 \%$ of cancer patients reported moderate to high levels of need for help dealing with FCR (Hartl, 2003; Hodgkinson, Butow, Hunt, Pendlebury, Hobbs, Lo, et al., 2007; Hodgkinson, Butow, Hunt, Pendlebury, Hobbs, \& Wain, 2007). A systematic review (Simard et al., 2013) of previous studies found that survivors diagnosed at a young age, female, and with higher education level were more likely to suffer FCR compared with their counterparts. Meta-analysis showed that having had a mastectomy (Koch, Jansen, Brenner, \& Arndt, 2013), radiotherapy (Yang, Cameron, \& Humphris, 2016) or chemotherapy (Yang, Wen, Bedi, \& Humphris, 2017) were strong predictors of higher FCR. Other demographic and clinical factors, such as marital status, employment, cancer stage, and treatment type were still conflictive.

Several measurements have been developed and utilized to assess FCR. Thewes and 
colleagues (Thewes et al., 2012) completed a review of existing FCR measurements in 2012, ranging from 2-item questionnaires to a 43-item questionnaire, and eventually they found 20 unique self-reported FCR assessment tools. Fear of Cancer Recurrence Inventory (FCRI) (Simard \& Savard, 2009) was one of the commonly used questionnaires. This multi-item scale has the strength of evaluating a variety of qualities/features of FCR. However, it can be burdensome to complete, timeconsuming and challenging to score and interpret (Humphris, Watson, Sharpe, \& Ozakinci, 2018). On the contrary, brief FCR questionnaire, such as the 2-item Fear of Recurrence Index (FRI) (Lasry \& Margolese, 1992), even though was very easy to administer, it showed serious psychometrical weakness. Thus, researchers argued that short uni-dimensional FCR measure could be considered as the main instrument for assessing and screening patients (Humphris et al., 2018).

In mainland China, only one FCR related scale has been translated and proved to be valid, that is, the Chinese version of the Fear of Progression Questionnaire-Short Form (FoP-Q-SF). In 2015, Wu et al. (Wu, 2015) investigated the reliability and validity of the Chinese FoP-Q-SF in 1031 liver cancer patients and confirmed that the scale was suitable for assessing FoP in Chinese cancer patients. However, no specific FCR instrument has been introduced in mainland China.

The main purpose of this study is to translate and evaluate the psychometric properties of the 7-item Fear of Cancer Recurrence (FCR-7) questionnaire. This scale is based upon a set of 7 questions that have been selected from extant measures within the literature to assess directly FCR (Humphris et al., 2018). It has already 
been utilized in numerous specific cancer populations, specifically in the UK (Rogers, Cross, Talwar, Lowe, \& Humphris, 2016). The Flesch readability index test showed that the FCR-7 was equivalent to 'Plain English, easily understood by thirteen- to fifteen-year-old students' (Humphris et al., 2018). In the current study, we aimed to: 1) translate the FCR-7 into Chinese and evaluate both the linguistic and cultural equivalence of the scale; 2 ) test its psychometric properties in a mixed group of Chinese cancer patients; 3 ) investigate the association of sociodemographic and clinical variables to FCR. 


\section{Methods}

\subsection{Participants and settings}

A cross-sectional study was used. All participants were consecutively recruited from the department of Radiotherapy and Oncology (Nanfang Hospital, Level III tertiary hospital) and Guangdong Cancer Center (Guangdong General Hospital, Level III tertiary hospital). Data were collected from $1^{\text {st }}$ January to $30^{\text {th }}$ July 2018 . Patients were eligible if they were: a) adults (above 18 years old); b) able to read, write and understand Mandarin or Cantonese; and c) with a cancer diagnosis. Excluded criteria were: a) patients who were blind/deaf; b) patients who had serious mental illness (i.e. schizophrenia); c) patients who received palliative treatment; or d) patients who had disturbance of consciousness.

Overall, 1153 eligible patients were invited to participate, and 1025 of them agreed (response rate $89 \%$ ). Of the 1025 participants, about $90 \%$ of the subjects were female and ranged in age from 20 to 90 years old (Mean $=48.29$ ). Most of the patients were married, living with family members, and with low education background and monthly income. Nearly $80 \%$ of the patients had been diagnosed with breast cancer and many of them had received surgery (91.3\%), chemotherapy (88.1\%) and radiotherapy (87.5\%).

\subsection{Instruments}

\subsubsection{Personal Information Sheet}

A study specific set of questions (demographic/clinical sheet) were formulated to assess patient's gender, age, marital, education, monthly income, employment 
status, cancer stage, treatment type, family cancer history, and self-rated physical morbidity. The last three were measured by simple 'Yes/No' questions: 1) Did you received surgery/chemotherapy/radiotherapy? 2) Do you have a family cancer history? 3) Do you suffer from any other physical comorbidity, such as diabetes, hypertension, musculo-skeletal, etc.? For those who were uncertain about their cancer stage/treatment type, data were recollected from their medical records.

\subsubsection{Fear of Cancer Recurrence (FCR-7) questionnaire}

The 7-item FCR was developed at the University of St Andrews, Scotland by Professor Humphris and his colleagues. It is used to assess patient's recurrence fears and has been used with patients with breast, colorectal and head and neck cancer in a variety of clinical centers in the UK (Rogers et al., 2016). The reliability of the questionnaire is good with an internal consistency of $0.92(95 \% \mathrm{Cl}: 0.90,0.94)$ and evidence for validity (Humphris et al., 2018). No cut-off has been reported other than the statistical $60^{\text {th }}($ score $=17)$ and $90^{\text {th }}($ score $=27)$ percentiles which have been regarded as levels for 'moderate' and 'high' reports of patient's FCR respectively.

\subsubsection{Fear of Progression Questionnaire-Short Form (FoP-Q-SF)}

The 12-item FoP-Q-SF is a short form of the original Fear of Progression Questionnaire (FoP-Q) (Herschbach et al., 2005). It has been utilized to samples of various cancer patients by many countries (Mehnert, Berg, Henrich, \& Herschbach, 2009; Mehnert, Herschbach, Berg, Henrich, \& Koch, 2006; Melchior et al., 2013). The items are scored on a 5 -point Likert scale (from $1=$ never, to $5=$ very often). The total score of the scale ranges from 12 to 60 , and higher total score indicates higher FoP. A 
score of 34 or above indicates a dysfunctional level of FoP (Herschbach et al., 2010). The psychometric properties of the Chinese FoP-Q-SF has been tested by Wu and her colleagues (Cronbach's alpha $=0.88)(\mathrm{Wu}, 2015)$, but no cut-offs for dysfunctional has been provided in the Chinese version scale.

\subsubsection{Patient Health Questionnaire (PHQ-9)}

The 9-item Patient Health Questionnaire is a commonly used screening tool for depression in medical settings. It evaluates the degree of depressive symptoms, and the items range from 0 to 3 ( $0=$ not at all, and $3=$ nearly everyday) (Herschbach et al., 2010). A total score of 5 or more indicates depressive symptoms, and higher total score indicates higher depression level. The Chinese PHQ-9 shows satisfactory psychometric properties (internal consistency $=0.89)($ Chen, 2015).

\subsubsection{General Anxiety Disorder Questionnaire (GAD-7)}

The 7-item General Anxiety Disorder Questionnaire is a brief self-report measurement used to evaluate person's anxiety symptoms. Response options are not at all, several days, more than half the days, and nearly every day, rated as $0,1,2$, and 3, respectively (Spitzer, Kroenke, Williams, \& Lowe, 2006). A total score of 5 or more indicates anxiety symptoms, and higher total score indicates higher anxiety level. The internal consistency of the Chinese GAD-7 is 0.91 (Zheng, 2013).

\subsection{Procedure}

\subsubsection{Translation}

The FCR-7 was translated according to the recommendations suggested by Bracken 
and Barona (Bracken BA, 1991). In this study, the first author (YY) translated the FCR7 from English to Chinese, then two bilingual translators (professors working at the Southern Medical University, both had experience of translating and validating instruments) who blinded to the FCR-7 were asked to complete the back-translation. To make sure the meaning of each item was kept, a comparison was made between the original and the re-translated English versions. Disagreements were discussed and agreed upon by the researcher and both the back-translators.

\subsubsection{Data collection}

Approval for the study was obtained from the hospital Research Ethics Committee (ref No: NFEC-2018-038). Patients were approached by three co-authors (SHW, WHM, and LWJ) who are all chief physicians. After patients who showed interest in participating were told about the purpose of the study, a written informed consent form was provided. All patients were given the option of allowing or refusing their involvement in the study and then were asked to complete a personal information sheet, the FCR-7, FoP-Q-SF, PHQ-9, GAD-7 and returned to the research staff immediately. The whole procedure was supervised by the last author (ZB) who is an experienced licensed psychiatrist.

\subsection{Data analysis}

All statistical analyses were calculated with SPSS v16 and STATA 15. Normal distribution of all item scores and the total score was tested using the KolmogorovSmirnow test. Item analysis was performed with calculation of means (M), standard deviations (SD), floor and ceiling effects. The items of the FCR-7 were tested with 
part-whole correlated Pearson correlations between item and scale value. Content equivalence was established by an expert panel. Internal consistency was determined with Cronbach's alpha, and bivariate correlations (Pearson's r) with the FoP-Q-SF, PHQ-9, and GAD-7 were used to investigate convergent validity. Descriptive statistics were used to analyze the demographic/clinical data. Difference in means were investigated using a t test for independent samples. Analysis of variance were used to identify group effects.

\section{Item analysis}

Floor and ceiling effects were indicated when one fourth (25\%)of the participants reported experiencing 'not at all' (floor effect) or 'all the time' (ceiling effect) (Fidika, Herle, Herschbach, \& Goldbeck, 2015). The item-scale value correlations were calculated using Pearson's correlation coefficients. Correlations above 0.40 are recommended, but correlations below 0.30 are usually considered unacceptably low (D. F. Polit \& Beck, 2009).

\section{Content equivalence}

Content equivalence implies that each item in the instrument has consistent cultural relevance (Flaherty et al., 1988). Content equivalence of the Chinese FCR-7 was examined by a panel of experts. The panel included three oncologists (WHM, LWJ, and SHW), two clinical psychologists (ZJY, ZB) and a psychology nurse specialist (LT). A four-point scale (from 1=not relevant at all, to $4=$ =very relevant) was completed by the experts to measure the relevancy of each item to the concept of FCR. The Content Validity Index (CVI), which indicates the percentage of the total items rated as either 
three or four, was calculated. A CVI score of $80 \%$ or higher is considered to indicate good content validity (Waltz, 1988).

Validity Test

Convergent validity was measured by Pearson's correlation to examine the association between scores on the FoP-Q-SF, PHQ-9, GAD-7 and FCR-7. The KaiserMeyer-Olkin test was conducted. The total sample was split randomly into two samples and EFA (using Horn's parallel analysis: 'paran' command in STATA) was performed on one sample, and CFA was performed on the remaining sample. The goodness-of-fit indexes used included: $\mathrm{X}^{2} /$ degrees of freedom (df) ratio, Comparative Fit Index (CFI), Root Mean Square Error of Approximation (RMSEA). The criteria for goodness-of-fit indexes are as followed: $X^{2} / d . f \leq 3, C F I \geq 0.95$, RMSEA $\leq 0.08$ (Li, Chung, Ho, Chiu, \& Lopez, 2013).

\section{Reliability Test}

Internal consistency reliability of the Chinese version FCR-7 was assessed by calculating the Cronbach's alpha. Internal consistency reliability is acceptable with a Cronbach's alpha above 0.70 , correlations of 0.80 and higher are highly desirable (D. F. Polit \& Beck, 2009). A third of the participants were randomly selected (using a random number generator) to respond to the FCR-7 again by telephone after 1 month, and test-retest reliability was assessed using Pearson's $r$ between the FCR-7 total score at initial assessment and 1-month reassessment. 


\section{Results}

\subsection{Participant Characteristics}

Patients who were older $(P<.001)$, full-time employed $(P=.005)$ and had been diagnosed with breast cancer $(P<.001)$ tended to report lower FCR. On the contrary, patients with low monthly salary $(P<.001)$, family cancer history $(P=.012)$, and those who had gone through chemotherapy $(P=.001)$ were more likely to experience higher FCR (table 1). One month later, 350 participants were invited by telephone to rate their FCR levels again and 285 of them completed the measurement (response rate 81\%). Table 2 shows the comparison of demographic and clinical characteristics between patients of the initial assessment and retest measurement. Significant group difference was found in age $(P=.037)$.

\subsection{Item Characteristics}

In the current study, floor effect was found for item 4 (33.4\%), and no ceiling effects were found. The item-total scale correlations ranged from 0.583 to 0.872 , which were all acceptable. Five out of seven items had high correlation (coefficients higher than 0.80 , table 3).

\subsection{Content Equivalence}

The total CVI was $88 \%$ (ranged from 63 to 100\%). The majority of the items were rated as quite or very relevant (score $=3$ or 4 ), with the exception of item 6 . Omitting item 6 the CVI was recalculated at 94\% (ranged from 88 to 100\%), which indicated that the content of most items reflected the underlying construct. 


\subsection{Validity Test}

The Kaiser-Meyer-Olkin test gave satisfactory high values of 0.91 for FCR-7, which meant sufficient variance to perform factor analysis. The EFA revealed single factor structure (eigenvalue for first factor $=4.26$ ). The second factor adjusted eigenvalue was 0.037 which was below the random derived parallel eigenvalue of 0.19 which was averaged over 50 replications (see Supplementary file). This demonstrated that there was no evidence for a substantial second factor that comprised sufficient meaningful variance over the calculated random variation. The factor loadings for all items were high (>0.7) with the exception of item 6 which was 0.578 (see table 3). The convergent validity of FCR-7 was assessed by calculating the correlations between FCR-7 total scores and the scores of FoP-Q-SF, PHQ-9 and GAD-7. Table 4 shows that the FCR-7 total score was significantly associated with the other three instruments ( $r$ ranged from 0.522 to 0.756 ). The fit indices for the CFA were supportive of a single unidimensional scale. The fit was demonstrated by a $X^{2} / d f$ fit index that was 1.79 and below recommended level of 3.0, CFI = 0.996; RMSEA = $0.039(95 \% \mathrm{Cl}: 0.01,0.07)$. These numerical values provided reassurance that the items behaved psychometrically as expected.

\subsection{Reliability Test}

The Cronbach's alpha of the scale was 0.87. Deletion of any item would not have indicated any improvements to internal consistency. As for the test-retest reliability, the Pearson's correlation coefficient between initial and one-month reassessment was 0.90 . 


\section{Discussion}

The main aim of this study was to translate a valid and reliable self-report scale for cancer recurrence fear, the FCR-7. Our results indicated that the Chinese version of the FCR-7 had satisfactory psychometric properties in Chinese cancer patients.

Reliability, which refers to the consistency between independent measurements of the same concept/phenomenon, was a prerequisite for a valid scale (D. E. Polit, 1997). Cronbach's alpha is commonly used to evaluate an instrument's reliability (Salkind, 2000). This study found that the Chinese FCR-7 scale has good internal consistency, with the Cronbach's alpha $=0.87$, which is slightly lower than the original scale that had been reported a coefficient of 0.92 (Humphris et al., 2018). Test-retest reliability with one-month interval was also assessed and considered satisfactory. However, we found that patients who completed reassessment were significantly younger than those at initial assessment. One possible reason is that younger participants were easier and more likely to be successfully reached by research staff because they used/answered their cell phones more frequently than older patients. Similar to earlier findings (Humphris et al., 2018), high item-total scale correlations (coefficients greater than 0.7 ) were found except for item 6. It is probably because item 6 focuses more on the behavioral response (self-examination behavior) to FCR while other items are describing the cognitive processing of FCR.

Construct validity was supported by correlations observed between total scores on the Chinese FCR-7 and other three relevant instruments (FoP-Q-SF, PHQ-9, and GAD7). Our study found a significant positive relationship between FCR-7, FoP, anxiety 
and depression scores. This finding is consistent with previous studies as researchers indicated that people with high levels of recurrence fears would tend to report more depressive, anxiety symptoms and psychological distress (Llewellyn, Weinman, McGurk, \& Humphris, 2008; Thewes et al., 2013). In addition, positive association between FCR and HADS (Hospital Anxiety and Depression Scale) was also found by previous reports (Hinz, Mehnert, Ernst, Herschbach, \& Schulte, 2015; Humphris et al., 2018; Simard, Savard, Gonthier, Tremblay, \& Maheux, 2005; Simard et al., 2013). In accordance with the original scale (Humphris et al., 2018), the EFA revealed single factor structure and the factor loadings for most items were satisfactory (greater than 0.7). CFA was conducted on the remaining half sample to examine the scale's factor structure more precisely, and the outcomes of the evaluation fit were all convincing, which confirmed a satisfactory fit between the hypothesized model and the data (Chan, Chow, \& Lo, 2005).

When compared with the Chinese FoP-Q-SF instrument, which is the only validated instrument measuring cancer patient's recurrence fear in mainland China, the Chinese version of FCR-7 demonstrated similar internal consistency (0.87 in FCR-7 versus 0.88 in FoP-Q- SF), better construct validity (CFI $=0.996, \mathrm{RMSEA}=0.039$ in FCR-7 versus $\mathrm{CFI}=0.902, \mathrm{RMSEA}=0.052$ in FoP-Q- SF), satisfactory content validity, and appropriate convergent validity.

In this study, we found that patients who were older and full-time employed tended to report lower FCR. These findings were consistent with several other FCR studies (Crist \& Grunfeld, 2013; Hartl, 2003; Simard et al., 2013). It is reasonable to assume 
that younger patients may consider their cancer as more unexpected (Simard et al., 2013) and patients who are unemployed/part-time employed are under greater economic burdens compared to those who have stable monthly income (Skaali et al., 2009). We also found that patients with a family cancer history were more likely to experience higher FCR. To our best knowledge, only one study reported family cancer history factor as a significant predictor of FCR (Dumalaon-Canaria, Prichard, Hutchinson, \& Wilson, 2016). Thus, the link between family history and FCR is still weak and further investigations are needed. Our result showed that breast cancer patients tended to have less fear. However, conflictive evidences were reported, for example, Simard (Simard et al., 2010) and Kornblith (Kornblith et al., 2007) observed higher FCR among breast cancer women, while others found no significant association between cancer site and FCR (Simard et al., 2013). The inconsistent result we found in this investigation might be explained by the uneven samples of the study - nearly $80 \%$ of participants were female breast cancer patients. Another possible reason is the 'cultural difference' between the eastern and western patients since an individual's illness perception might be influenced by the cultural system where they are located. Further studies with more male and mixed samples are needed.

The strengths of this study are the relatively large sample size and inclusion of different cancer diagnoses. However, there are a number of limitations that should be acknowledged. First, nearly $80 \%$ of the participants are breast cancer patients and about $90 \%$ of them are female. A potential sample bias may exist because of the over-representation of female breast cancer patients and the small subsample of male participants may limit representativeness of our results. Second, only 2 
southern hospitals were involved in this study, cancer centers in the middle and northern part of China were not included. The psychometric testing may be limited by the use of convenience sampling and the fact that the data recruitment sites are located in only one single city. Third, discriminant validity of the scale has not been examined in the current study. Further studies may consider investigating the correlations between scores on FCR-7 and health-related quality of life, psychological/social functioning, or overall well-being. A negative correlation may support the discriminant validity of the FCR-7. Fourth, many other important variables, such as time since cancer diagnosis, and surgery type were not examined in this study. Last but not least, cut-offs for dysfunctional/clinical significance of the Chinese FCR-7 have not been defined yet. 


\section{Conclusion}

The FCR-7 has been translated and successfully culturally adapted into a Chinese version. The scale demonstrated robust psychometric properties, suggesting that it is a reliable and valid measurement for assessing patient's recurrence fear and may be considered to widely use in clinical service in mainland China. More validated Chinese instruments of FCR with clear cut-off values should be introduced and utilized in the future. 


\section{Reference}

Avis, N. E., Smith, K. W., McGraw, S., Smith, R. G., Petronis, V. M., \& Carver, C. S. (2005). Assessing quality of life in adult cancer survivors (QLACS). Qual Life Res, 14(4), 1007-1023.

Bracken BA, B. A. (1991). State of the art procedures for translating, validating and using psychoeducational tests in cross-cultural assessment. Sch Psychol Int, 12, 119-132.

Chan, L. F., Chow, S. M., \& Lo, S. K. (2005). Preliminary validation of the Chinese version of the Pediatric Quality of Life Inventory. Int J Rehabil Res, 28(3), 219-227.

Chen, M., Sheng, L., Qu, s. (2015). Diagnostic test of screening depressive disorder in general hospital with the Patient Health Questionnaire (in Chinese). Chinese Mental Health, 29(4), 241-245.

Cohee, A. A., Adams, R. N., Johns, S. A., Von Ah, D., Zoppi, K., Fife, B., . . Champion, V. L. (2015). Longterm fear of recurrence in young breast cancer survivors and partners. Psychooncology. doi:10.1002/pon.4008

Crist, J. V., \& Grunfeld, E. A. (2013). Factors reported to influence fear of recurrence in cancer patients: a systematic review. Psychooncology, 22(5), 978-986. doi:10.1002/pon.3114

Dumalaon-Canaria, J. A., Prichard, I., Hutchinson, A. D., \& Wilson, C. (2016). Fear of cancer recurrence and psychological well-being in women with breast cancer: The role of causal cancer attributions and optimism. Eur J Cancer Care (Engl). doi:10.1111/ecc.12579

Fidika, A., Herle, M., Herschbach, P., \& Goldbeck, L. (2015). Fear of disease progression questionnaire for parents: Psychometric properties based on a sample of caregivers of children and adolescents with cystic fibrosis. J Psychosom Res, 79(1), 49-54. doi:10.1016/j.jpsychores.2015.02.014

Flaherty, J. A., Gaviria, F. M., Pathak, D., Mitchell, T., Wintrob, R., Richman, J. A., \& Birz, S. (1988). Developing instruments for cross-cultural psychiatric research. J Nerv Ment Dis, 176(5), 257263.

Hartl, K. (2003). Impact of medical and demographic factors on long-term quality of life and body image of breast cancer patients. Annals of Oncology, 14(7), 1064-1071. doi:10.1093/annonc/mdg289

Herschbach, P., Berg, P., Dankert, A., Duran, G., Engst-Hastreiter, U., Waadt, S., . . Henrich, G. (2005). Fear of progression in chronic diseases: psychometric properties of the Fear of Progression Questionnaire. J Psychosom Res, 58(6), 505-511. doi:10.1016/j.jpsychores.2005.02.007

Herschbach, P., Berg, P., Waadt, S., Duran, G., Engst-Hastreiter, U., Henrich, G., . . Dinkel, A. (2010). Group psychotherapy of dysfunctional fear of progression in patients with chronic arthritis or cancer. Psychother Psychosom, 79(1), 31-38. doi:10.1159/000254903

Hinz, A., Mehnert, A., Ernst, J., Herschbach, P., \& Schulte, T. (2015). Fear of progression in patients 6 months after cancer rehabilitation-a- validation study of the fear of progression questionnaire FoP-Q-12. Support Care Cancer, 23(6), 1579-1587. doi:10.1007/s00520-0142516-5

Hodgkinson, K., Butow, P., Hunt, G. E., Pendlebury, S., Hobbs, K. M., Lo, S. K., \& Wain, G. (2007). The development and evaluation of a measure to assess cancer survivors' unmet supportive care needs: the CaSUN (Cancer Survivors' Unmet Needs measure). Psychooncology, 16(9), 796804. doi:10.1002/pon.1137

Hodgkinson, K., Butow, P., Hunt, G. E., Pendlebury, S., Hobbs, K. M., \& Wain, G. (2007). Breast cancer survivors' supportive care needs 2-10 years after diagnosis. Support Care Cancer, 15(5), $515-$ 


\section{3. doi:10.1007/s00520-006-0170-2}

Humphris, G. M., Watson, E., Sharpe, M., \& Ozakinci, G. (2018). Unidimensional scales for fears of cancer recurrence and their psychometric properties: the FCR4 and FCR7. Health Qual Life Outcomes, 16(1), 30. doi:10.1186/s12955-018-0850-x

Koch, L., Jansen, L., Brenner, H., \& Arndt, V. (2013). Fear of recurrence and disease progression in longterm (>/= 5 years) cancer survivors--a systematic review of quantitative studies.

Psychooncology, 22(1), 1-11. doi:10.1002/pon.3022

Kornblith, A. B., Powell, M., Regan, M. M., Bennett, S., Krasner, C., Moy, B., . . W Winer, E. (2007). Longterm psychosocial adjustment of older vs younger survivors of breast and endometrial cancer. Psychooncology, 16(10), 895-903. doi:10.1002/pon.1146

Lasry, J. C., \& Margolese, R. G. (1992). Fear of recurrence, breast-conserving surgery, and the trade-off hypothesis. Cancer, 69(8), 2111-2115.

Lebel, S., Ozakinci, G., Humphris, G., Mutsaers, B., Thewes, B., Prins, J., . . University of Ottawa Fear of Cancer Recurrence Colloquium, a. (2016). From normal response to clinical problem: definition and clinical features of fear of cancer recurrence. Support Care Cancer, 24(8), 32653268. doi:10.1007/s00520-016-3272-5

Lebel, S., Rosberger, Z., Edgar, L., \& Devins, G. M. (2009). Emotional distress impacts fear of the future among breast cancer survivors not the reverse. J Cancer Surviv, 3(2), 117-127. doi:10.1007/s11764-009-0082-5

Li, H. C., Chung, O. K., Ho, K. Y., Chiu, S. Y., \& Lopez, V. (2013). Effectiveness of an integrated adventurebased training and health education program in promoting regular physical activity among childhood cancer survivors. Psychooncology, 22(11), 2601-2610. doi:10.1002/pon.3326

Llewellyn, C. D., Weinman, J., McGurk, M., \& Humphris, G. (2008). Can we predict which head and neck cancer survivors develop fears of recurrence? J Psychosom Res, 65(6), 525-532. doi:10.1016/j.jpsychores.2008.03.014

Mehnert, A., Berg, P., Henrich, G., \& Herschbach, P. (2009). Fear of cancer progression and cancerrelated intrusive cognitions in breast cancer survivors. Psychooncology, 18(12), 1273-1280. doi:10.1002/pon.1481

Mehnert, A., Herschbach, P., Berg, P., Henrich, G., \& Koch, U. (2006). [Fear of progression in breast cancer patients--validation of the short form of the Fear of Progression Questionnaire (FoPQ-SF)]. Z Psychosom Med Psychother, 52(3), 274-288.

Melchior, H., Buscher, C., Thorenz, A., Grochocka, A., Koch, U., \& Watzke, B. (2013). Self-efficacy and fear of cancer progression during the year following diagnosis of breast cancer. Psychooncology, 22(1), 39-45. doi:10.1002/pon.2054

Polit, D. E. (1997). Essentials of Nursing Research Methods Appraisal and Utilization, 4th edn. Philadelphia, PA: Lippin-cott Raven Publishers.

Polit, D. F., \& Beck, C. T. (2009). International gender bias in nursing research, 2005-2006: a quantitative content analysis. Int J Nurs Stud, 46(8), 1102-1110. doi:10.1016/j.ijnurstu.2009.02.002

Rogers, S. N., Cross, B., Talwar, C., Lowe, D., \& Humphris, G. (2016). A single-item screening question for fear of recurrence in head and neck cancer. Eur Arch Otorhinolaryngol, 273(5), 1235-1242. doi:10.1007/s00405-015-3585-x

Salkind, N. J. (2000). Statistics for People Who Hate Statistics. Thousand Oaks, CA.: Sage Publications, Inc. 
Simard, S., \& Savard, J. (2009). Fear of Cancer Recurrence Inventory: development and initial validation of a multidimensional measure of fear of cancer recurrence. Support Care Cancer, 17(3), 241-251. doi:10.1007/s00520-008-0444-y

Simard, S., Savard, J., Gonthier, C., Tremblay, V., \& Maheux, J. (2005). Development and empirical validation of the fear of cancer recurrence inventory. Psycho-Oncology, 14(1), S51-S51.

Simard, S., Savard, J., \& Ivers, H. (2010). Fear of cancer recurrence: specific profiles and nature of intrusive thoughts. J Cancer Surviv, 4(4), 361-371. doi:10.1007/s11764-010-0136-8

Simard, S., Thewes, B., Humphris, G., Dixon, M., Hayden, C., Mireskandari, S., \& Ozakinci, G. (2013). Fear of cancer recurrence in adult cancer survivors: a systematic review of quantitative studies. J Cancer Surviv, 7(3), 300-322. doi:10.1007/s11764-013-0272-z

Skaali, T., Fossa, S. D., Bremnes, R., Dahl, O., Haaland, C. F., Hauge, E. R., . . Dahl, A. A. (2009). Fear of recurrence in long-term testicular cancer survivors. Psychooncology, 18(6), 580-588. doi:10.1002/pon.1437

Spitzer, R. L., Kroenke, K., Williams, J. B., \& Lowe, B. (2006). A brief measure for assessing generalized anxiety disorder: the GAD-7. Arch Intern Med, 166(10), 1092-1097. doi:10.1001/archinte.166.10.1092

Thewes, B., Bell, M. L., Butow, P., Beith, J., Boyle, F., Friedlander, M., . . Members of the, F. C. R. S. A. C. (2013). Psychological morbidity and stress but not social factors influence level of fear of cancer recurrence in young women with early breast cancer: results of a cross-sectional study. Psychooncology, 22(12), 2797-2806. doi:10.1002/pon.3348

Thewes, B., Butow, P., Zachariae, R., Christensen, S., Simard, S., \& Gotay, C. (2012). Fear of cancer recurrence: a systematic literature review of self-report measures. Psychooncology, 21(6), 571-587. doi:10.1002/pon.2070

Waltz, C. F. (1988). Educational outcomes: assessment of quality--a prototype for student outcome measurement in nursing programs. NLN Pub/(18-2255), i-viii, 1-36.

Wu, Q., Ye, Z., Li, L., Liu, P. (2015). Reliability and validity of Chinese version of Fear of Progression Questionnaire-Short Form for cancer patients (in Chinese). Chin J Nurs, 12(12), 1515-1519.

Yang, Y., Cameron, J., \& Humphris, G. (2016). The relationship between cancer patient's fear of recurrence and radiotherapy: a systematic review and meta-analysis. Psychooncology. doi:10.1002/pon.4224

Yang, Y., Wen, Y., Bedi, C., \& Humphris, G. (2017). The relationship between cancer patient's fear of recurrence and chemotherapy: A systematic review and meta-analysis. J Psychosom Res, 98, 55-63. doi:10.1016/j.jpsychores.2017.05.002

Zheng, Q. (2013). Reliability and validity of Chinese version of Generalized Anxiety Disorder 7-item (GAD-7) scale in screening anxiety disorder in outpatients from traditional Chinese internal department (in Chinese). Chinese Mental Health, 27(3), 163-168. 
Table 1: Characteristics of Participants at Initial Assessment ( $n=1025)$

\begin{tabular}{|c|c|c|c|c|}
\hline Sociodemographic & $\mathrm{n}(\%)$ & FCR (M $\pm S D)$ & Statistics & $P$ value \\
\hline \multicolumn{5}{|l|}{ Gender } \\
\hline Male & $106(10.3)$ & $20.8 \pm 7.4$ & & \\
\hline Female & 919 (89.7) & $20.0 \pm 6.3$ & $\mathrm{t}=1.13$ & .259 \\
\hline \multicolumn{5}{|l|}{ Age } \\
\hline Age below 35 & $146(14.2)$ & $20.6 \pm 6.5$ & & \\
\hline Age between 35-60 & 675 (65.9) & $21.8 \pm 7.0$ & & \\
\hline Age above 60 & 204 (19.9) & $19.4 \pm 6.1$ & $\mathrm{~F}=12.21$ & $<.001$ \\
\hline \multicolumn{5}{|l|}{ Marital Status } \\
\hline Single & $72(7.0)$ & $20.6 \pm 6.6$ & & \\
\hline Married & $879(85.8)$ & $20.1 \pm 6.4$ & & \\
\hline Divorced & 40 (3.9) & $18.3 \pm 6.5$ & & \\
\hline Widowed & $34(3.3)$ & $20.1 \pm 6.9$ & $F=1.16$ & .324 \\
\hline \multicolumn{5}{|l|}{ Education Level } \\
\hline High School or below & $682(66.5)$ & $20.2 \pm 6.6$ & & \\
\hline Undergraduate & $261(25.5)$ & $19.6 \pm 5.9$ & & \\
\hline Postgraduate or above & $82(8.0)$ & $20.5 \pm 6.8$ & $F=1.07$ & .342 \\
\hline \multicolumn{5}{|l|}{ Living Arrangement } \\
\hline Living alone & $46(4.5)$ & $20.7 \pm 7.0$ & & \\
\hline Living with Family & $955(93.2)$ & $20.1 \pm 6.4$ & & \\
\hline Living with Friends & $24(2.3)$ & $17.5 \pm 6.1$ & $F=2.12$ & .120 \\
\hline \multicolumn{5}{|l|}{ Monthly Salary (Yuan) } \\
\hline Less than 3000 & $474(46.2)$ & $21.1 \pm 6.7$ & & \\
\hline $3000-5000$ & $267(26.0)$ & $19.4 \pm 6.1$ & & \\
\hline $5000-10000$ & $202(19.7)$ & $18.9 \pm 6.0$ & & \\
\hline More than 10000 & $82(8.0)$ & $19.1 \pm 5.6$ & $F=8.02$ & $<.001$ \\
\hline \multicolumn{5}{|l|}{ Employment } \\
\hline Full time & $376(36.7)$ & $19.1 \pm 5.7$ & & \\
\hline Part time & $55(5.4)$ & $20.0 \pm 5.8$ & & \\
\hline Unemployment & 306 (29.9) & $20.8 \pm 6.8$ & & \\
\hline Retired & $288(28.1)$ & $20.4 \pm 6.7$ & $F=4.29$ & .005 \\
\hline \multicolumn{5}{|l|}{ Cancer Site } \\
\hline Breast Cancer & $803(78.3)$ & $19.8 \pm 6.2$ & & \\
\hline Lung Cancer & $109(10.6)$ & $20.0 \pm 7.1$ & & \\
\hline Colorectal Cancer & $84(8.2)$ & $21.5 \pm 6.7$ & & \\
\hline Nasopharynx Cancer & $29(2.8)$ & $24.2 \pm 7.1$ & $F=6.35$ & $<.001$ \\
\hline \multicolumn{5}{|l|}{ Cancer Stage } \\
\hline Stage 1 & $69(6.7)$ & $20.1 \pm 6.4$ & & \\
\hline Stage 2 & $352(34.3)$ & $20.5 \pm 6.7$ & & \\
\hline Stage 3 & $524(51.1)$ & $19.7 \pm 6.2$ & & \\
\hline Stage 4 & $80(7.8)$ & $20.1 \pm 6.8$ & $F=1.03$ & .380 \\
\hline \multicolumn{5}{|l|}{ Chemotherapy } \\
\hline Yes & $903(88.1)$ & $20.3 \pm 6.3$ & & \\
\hline No & $122(11.9)$ & $18.2 \pm 6.7$ & $t=-3.36$ & .001 \\
\hline
\end{tabular}




\begin{tabular}{lcccc} 
Yes & $897(87.5)$ & $20.2 \pm 6.3$ & & \\
No & $128(12.5)$ & $19.3 \pm 6.6$ & $t=-1.47$ & .142 \\
$\begin{array}{l}\text { Surgery } \\
\text { Yes }\end{array}$ & $936(91.3)$ & $20.1 \pm 6.5$ & & \\
No & $89(8.7)$ & $19.1 \pm 5.4$ & $\mathrm{t}=-1.78$ & .078 \\
Cancer Family History & & & & \\
Yes & $260(25.4)$ & $20.9 \pm 6.3$ & & \\
No & $765(74.6)$ & $19.8 \pm 6.3$ & $\mathrm{t}=-2.53$ & .012 \\
$\begin{array}{l}\text { Physical Comorbidity } \\
\text { Yes }\end{array}$ & $671(65.5)$ & $19.6 \pm 6.3$ & & \\
No & $354(34.5)$ & $20.8 \pm 6.6$ & $\mathrm{t}=2.77$ & $\mathbf{0 0 6}$ \\
\hline Abbreviation: M: mean; SD: standard deviation; & & &
\end{tabular}


Table 2: Comparison of Subjects at Initial and Retest Assessment

\begin{tabular}{|c|c|c|c|c|}
\hline Characteristics & $\begin{array}{l}\text { Initial test } \\
(n=1025)\end{array}$ & $\begin{array}{l}\text { Re-test } \\
(\mathrm{n}=285)\end{array}$ & $t / x^{2}$ & $\boldsymbol{P}$ \\
\hline Age (year) & $48.29 \pm 11.88$ & $46.7 \pm 11.1$ & 2.09 & .037 \\
\hline Gender (male/female) \% & $10.3 / 89.7$ & 7.0/93.0 & 2.83 & .092 \\
\hline $\begin{array}{l}\text { Marital State (single/married } \\
\text { /divorced/widowed) \% }\end{array}$ & 7.0/85.8/3.9/3.3 & $6.0 / 87.4 / 2.8 / 3.9$ & 1.36 & .716 \\
\hline $\begin{array}{l}\text { Education (high school and below } \\
\text { /Undergraduate/Postgraduate and above) \% }\end{array}$ & $66.5 / 25.5 / 8.0$ & $63.9 / 30.1 / 6.0$ & 3.33 & .189 \\
\hline Living arrangement (alone/family/friend) \% & $4.5 / 93.2 / 2.3$ & $5.6 / 89.5 / 4.9$ & 6.01 & .051 \\
\hline Employment (full time/part time/unemployed/retired) \% & $36.7 / 5.4 / 29.9 / 28.1$ & $39.6 / 6.7 / 28.8 / 24.9$ & 2.11 & .550 \\
\hline Cancer site (breast/lung/colorectal/nasopharynx) \% & $78.3 / 10.6 / 8.2 / 2.8$ & $80.4 / 9.8 / 5.3 / 4.6$ & 4.88 & .181 \\
\hline Surgery (Yes/no) \% & $91.3 / 8.7$ & $90.5 / 9.5$ & 0.17 & .678 \\
\hline Chemotherapy (Yes/no) \% & $88.1 / 11.9$ & $84.9 / 15.1$ & 2.06 & .152 \\
\hline Radiotherapy (Yes/no) \% & $87.5 / 12.5$ & $85.6 / 14.4$ & 0.72 & .398 \\
\hline Comorbidity (Yes/no) \% & $65.5 / 34.5$ & $67.0 / 33.0$ & 0.24 & .625 \\
\hline Family Cancer History (Yes/no) \% & $25.4 / 74.6$ & $25.6 / 74.4$ & 0.01 & .932 \\
\hline
\end{tabular}


Table 3. Item Characteristics of the Chinese version FCR-7 ( $N=1025)$

\begin{tabular}{|c|c|c|c|c|c|c|}
\hline Items & M & SD & $\%$ Not at all & $\begin{array}{l}\text { \% All the } \\
\text { time }\end{array}$ & $r^{a}$ & loadings \\
\hline Q1: I am afraid that my cancer may recur & 2.97 & 1.06 & $8.8 \%$ & $10.9 \%$ & $.843^{* *}$ & .868 \\
\hline $\begin{array}{l}\text { Q2: I am worried or anxious about the possibility of cancer } \\
\text { recurrence }\end{array}$ & 2.79 & 1.01 & $9.6 \%$ & $7.1 \%$ & $.872 * *$ & .896 \\
\hline $\begin{array}{l}\text { Q3: How often have you worried about the possibility of getting } \\
\text { cancer again }\end{array}$ & 2.70 & 0.88 & $6.5 \%$ & $3.8 \%$ & $.846 * *$ & .890 \\
\hline Q4: I get waves of strong feelings about the cancer coming back & 2.04 & 0.97 & $33.4 \%$ & $2.7 \%$ & $.774 * *$ & .775 \\
\hline Q5: I think about the cancer returning when I didn't mean to & 2.44 & 0.91 & $15.3 \%$ & $2.6 \%$ & $.804^{* *}$ & .820 \\
\hline Q6: I examine myself to see if I have physical signs of cancer & 2.93 & 0.95 & $7.1 \%$ & $4.1 \%$ & $.583^{* *}$ & .578 \\
\hline $\begin{array}{l}\text { Q7: To what extent does worry about getting cancer again spill } \\
\text { over or intrude on your thoughts and activities }\end{array}$ & 4.19 & 2.23 & $12.7 \%$ & $1.0 \%$ & $.829 * *$ & .749 \\
\hline
\end{tabular}

Note: validation data is based on scale in Chinese. ${ }^{* *} \mathrm{P}<.01$; a: item-total scale correlation; 
Table 4. Correlations of the FCR-7 Total Score with FoP-Q-SF, PHQ-9 and GAD-7.

\begin{tabular}{llllll}
\hline & $\mathrm{M} \pm \mathrm{SD}$ & $\mathrm{FCR}-7$ & FoP-Q-SF & PHQ-9 & GAD-7 \\
\hline FCR-7 & $20.05 \pm 6.41$ & 1 & $.756^{* *}$ & $.522^{* *}$ & $.553^{* *}$ \\
FoP-Q-SF & $29.78 \pm 7.93$ & & 1 & $.573^{* *}$ & $.551^{* *}$ \\
PHQ-9 & $5.13 \pm 4.92$ & & & 1 & $.811^{* *}$ \\
GAD-7 & $3.82 \pm 4.29$ & & & & 1 \\
\hline
\end{tabular}

${ }^{* *} P<.01$, M: mean; SD: standard deviation; FoP-Q-SF: Fear of Progression Questionnaire-Short Form; PHQ-9: Patient Health Questionnaire; GAD-7: General Anxiety Disorder Questionnaire; 


\section{Supplementary File: Horn's Parallel Analysis}

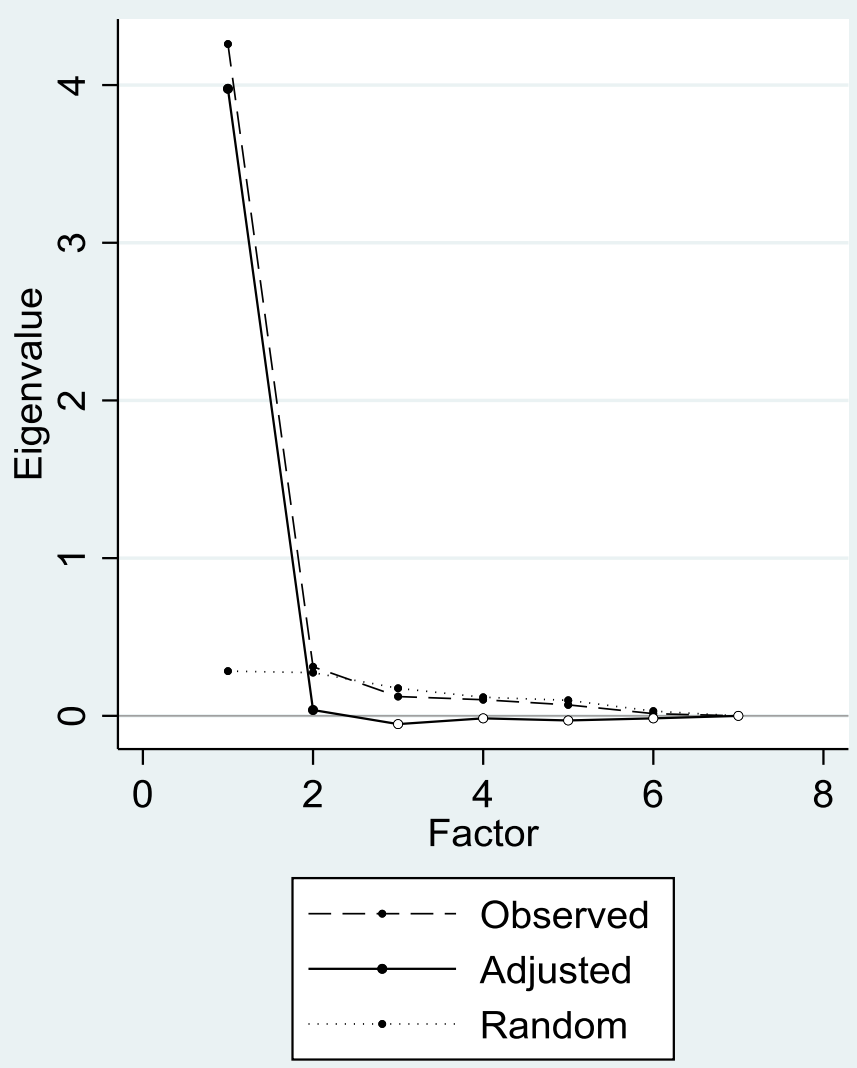

Note: The Horn's Parallel Analysis showing eigenvalues for the 7 potential factors in the the FCR-7 with the random data points plotted in comparison with the adjusted. The crossover of the adjusted values between factors 1 and 2 and the randomly derived values from 50 averaged replications provides evidence of a strong uni-dimensional measurement structure for the Chinese FCR-7 scale. 